\title{
Utilidad del [99m] Tc TRODAT-1 en el diagnóstico de la enfermedad de Parkinson
}

\author{
Utility of (99m) Tc TRODAT-1 imaging in \\ diagnosing Parkinson's disease
}

\author{
Johanna Ortiz • Bogotá, D.C. Steven Anthony Newman • EUA. \\ Sinay Arévalo, Héctor Fabio Restrepo • Bogotá, D.C.
}

\section{Resumen}

Propósito de la revisión: la enfermedad de Parkinson (EP) es un trastorno degenerativo caracterizado clínicamente por presentar temblor en reposo, rigidez y bradicinesia. El propósito es determinar la utilidad de la molécula TRODAT-1 en el diagnóstico de la EP.

Metodología: se realizó una búsqueda en las bases de datos: PUBMED, COCHRANE, MEDLINE, LILACS y SCIELO en un periodo de 10 años desde enero de 1998 a enero de 2008. Se obtuvieron 26 artículos, éstos se analizaron y se seleccionaron 10, de los cuales sólo seis respondían a las necesidades del estudio, de acuerdo con los criterios de inclusión. De los seis artículos analizados, cuatro fueron clasificados como evidencia grado (+) y los dos restantes evidencia grado (-) de acuerdo con las guías NICE. Todos los artículos revisados reportan una disminución importante en la captación del TRODAT-1 a nivel estriatal, su utilidad en el diagnóstico de EP en estadios tempranos, bajo costo y seguridad. Sólo tres reportan valores de sensibilidad y especificidad, pero su nivel de calidad no permite hacer una comparación de los mismos.

Conclusiones: se propone realizar estudios de prueba diagnóstica comparados con el diagnóstico clínico de la enfermedad, que tengan un acuerdo en la forma de plantear las mediciones semicuantitativas de las unidades de captación utilizando las mismas fórmulas para hacerlos comparables (Acta Med Colomb 2010; 35: 119-125).

Palabras clave: enfermedad de Parkinson, enfermedad de Parkinson idiopática, enfermedad de Parkinson con cuerpos de Lewy, parálisis agitante, $99 m$ Tc TRODAT-1, SPECT, tomografía computarizada de emisión de fotón simple, TC de emisión de fotón único.

\footnotetext{
Abstract

Purpose: Parkinson disease (PD) is a degenerative disorder. The clinical symptoms of this disease are resting tremor, rigidity and bradykinesia. The purpose of this literature review is to determine the utility of TRODAT -1 in the diagnosis of PD. A search covered PUBMED, COCHRANE, MEDLINE, LILACS and SCIELO databases, between January 1998 and January 2008.

Study selection: twenty six articles were obtained with their respective abstracts. After the first review, ten of them were chosen and only six of them met the inclusion criteria. Four articles were classified as grade evidence (+) and two as grade evidence (-) according to NICE guides. All the articles reviewed report significantly decreased striatal uptake of TRODAT-1 in early PD patients, suggesting that this is a useful, safe, and inexpensive tool in the diagnosis of early PD. It was not possible to perform a meta-analysis with the chosen articles because only three of them reported sensitivity and specificity, and each of these used different criteria for their semi-quantitative analyses. This variability makes comparison of the semiquantitative uptake criteria impossible.

Conclusions: establishment of a universal technique for quantitation of TRODAT-1 uptake is necessary in order to make meta-analysis viable and allow comparison of the usefulness of this agent among large numbers of patients and multiple populations (Acta Med Colomb 2010; 35: 119-125).

Keywords: Parkinson disease, parakysis agitans, Lewy bodies parkinson disease, 99m Tc TRODAT-1, SPECT, Single-Photon Emission-Computed Tomography, Radionuclide Tomography, Single-Photon Emission-Computed.
}

Dra. Johanna Ortiz Jiménez: Residente de Radiología e Imágenes Diagnósticas. Universidad del Rosario. Fundación Cardioinfantil - Instituto de Cardiología, Bogotá, D.C.; Dr. Steven Anthony Newman: Residente de Radiología e Imágenes Diagnósticas. Universidad Médica de Georgia, Augusta GA (EUA); Dr. Sinay Arévalo: Médico Nuclear. Fundación Cardioinfantil, Instituto de Cardiología, Bogotá, D.C.; Dr. Héctor Fabio Restrepo: Médico Epidemiólogo. Fundación Cardioinfantil, Instituto de Cardiología, Bogotá, D.C..

Este trabajo no ha sido presentado previamente en congresos o publicaciones. No existen conflictos de tipo financiero, ni de interés actuales o potenciales relacionados con este manuscrito.

Correspondencia. Dra. Johanna Ortiz Jiménez MD. Calle 163 A 13B - 60 - Tel. 571 6672727 Ext.1118. Bogotá, D.C.,Colombia E-mail: ortizj.johanna@ur.edu.co Recibido: 20/I/10 Aceptado: 02/IX/10 


\section{Introducción}

La EP es un trastorno degenerativo que se caracteriza clínicamente por presentar temblor en reposo, rigidez, bradicinesia e inestabilidad postural. Su diagnóstico es clasificado como posible, probable y definitivo teniendo en cuenta los criterios enumerados en la Tabla 1 (1). La EP tiene una prevalencia global de $0.2 \%$ que incrementa con la edad, afectando a $0.5 \%-1 \%$ de las personas entre 65 y 69 años y hasta el $3 \%$ de las personas mayores de 80 años. (2) Los síntomas clínicos típicos se presentan en personas que tienen una pérdida neuronal en la sustancia nigra de por lo menos el 50\%, siendo su diagnóstico tardío.

La medicina nuclear hace parte de las neuroimágenes moleculares, y pretende crear una molécula que se comporte en forma similar a las sustancias involucradas en la sinapsis dopaminérgica que tiene lugar en el cuerpo estríado, adicionando un radionúclido que pueda ser detectado por la gammacamára (3). Muchas sustancias han sido utilizadas entre ellas el ${ }^{18} \mathrm{~F}$ DOPA (dihidroxifenilalanina), ${ }^{11} \mathrm{C}$ metilfenidato y el ${ }^{99 m}$ Tc TRODAT-1; este último hace parte de la familia de los tropanos y se comporta como un transportador de la dopamina, está localizado en la hendidura sináptica y es necesario para regular la síntesis de este neurotransmisor (4).

El diagnóstico clínico probable de la EP se obtiene cuando el paciente ha perdido por lo menos el $50 \%$ de la población neuronal dopaminérgica, y las terapias neuroprotectoras ya no son tan eficaces en ese momento. Sí es posible mediante la utilización del TRODAT-1 realizar un diagnóstico temprano de la enfermedad, antes de una pérdida neuronal tan marcada, probablemente el paciente pueda beneficiarse de una terapéutica adecuadamente dirigida y enfocada a su enfermedad desde el inicio de la misma y en un futuro de terapéutica preventiva y medicamentos neuroprotectores, disminuyendo la carga psicológica que conlleva este tipo de desorden, la discapacidad que puede preverse y mejorando el pronóstico de la enfermedad.

\section{Selección de los estudios}

Se realizó una búsqueda y selección de los estudios controlados que involucraban la utilización del TRODAT-1 en el diagnóstico de la EP comparándolos con el diagnóstico clínico, publicados en un periodo de 11 años comprendido desde el 01 de enero de 1998 hasta el 01 de diciembre de 2009. La búsqueda fue realizada en las siguientes bases de datos: COCHRANE, PUBMED, MEDLINE, LILACS, SCIELO, OVID, PROQUEST MEDICAL, PROQUEST SCIENCE, PROQUEST HEALTH, EBSCO ACADEMIC, EBSCO MEDLINE FULL TEXT, utilizando combinaciones de los siguientes palabras "technetium Tc 99m TRODAT-1 "y términos MeSH: "Parkinson Disease", "Parkinson disease/diagnosis", ,"randomized controlled trial", "Clinical Trial, Meta-Analysis", "Practice Guideline", "Randomized Controlled Trial", "Clinical Trial, Phase IV", "Parkinsonian Disorders/radionuclide imaging", con los límites de idioma español o inglés. Esta búsqueda fue realizada a través de
Tabla 1. Criterios diagnósticos en EP (1).

\begin{tabular}{|c|}
\hline $\begin{array}{l}\quad \text { GRUPO A: Síntomas característicos de la EP } \\
\text { - Temblor en reposo. } \\
\text { - Bradicinesia. } \\
\text { - Rigidez. } \\
\text { - Inicio asimétrico. }\end{array}$ \\
\hline $\begin{array}{l}\text { GRUPO B: Síntomas sugestivos de diagnóstico alterno } \\
\text { - Características inusuales tempranas en el curso clínico. } \\
\text { - Inestabilidad postural prominente en los tres primeros años después del inicio } \\
\text { de los síntomas. } \\
\text { - Congelamiento en los tres primeros años. } \\
\text { - Alucinaciones relacionadas con el medicamento en los tres primeros años. } \\
\text { - Demencia que precede los síntomas motores en el primer año. } \\
\text { - Parálisis supranuclear (Otra que no sea la restricción a la supraversión). } \\
\text { - Disautonomía sintomática, severa no relacionada con medicamentos. } \\
\text { conectada con los síntomas del paciente (lesiones cerebrales focales, o uso de } \\
\text { neurolépticos en los últimos seis meses). }\end{array}$ \\
\hline $\begin{array}{l}\text { - Criterios para un diagnóstico POSIBLE de EP } \\
\text { Presencia de } 2 \text { de } 4 \text { criterios del grupo A, y al menos } 1 \text { de éstos es temblor o } \\
\text { bradicinesia } \\
\text { Además: } \\
\text { - Ninguno de los criterios del grupo B está presente. } \\
\text { - Los síntomas han estado presentes por menos de tres años, y ninguno de los } \\
\text { criterios del grupo B está presente en el momento. } \\
\text { - Se ha documentado una respuesta sustancial y sostenida a L dopa o a un } \\
\text { agonista dopaminérgico. } \\
\text { - El paciente no ha tenido un ensayo adecuado con L dopa o un agonista } \\
\text { dopaminérgico. }\end{array}$ \\
\hline $\begin{array}{l}\text { Presencia de } 2 \text { de } 4 \text { criterios del grupo A, y al menos } 1 \text { de estos es temblor o } \\
\text { bradicinesia } \\
\text { Además: } \\
\text { - Ninguno de los criterios del grupo B está presente. } \\
\text { - Los síntomas han estado presentes por menos de tres años, y ninguno de los } \\
\text { criterios del grupo B está presente en el momento. } \\
\text { - Se ha documentado una respuesta sustancial y sostenida a L dopa o a un } \\
\text { agonista dopaminérgico. } \\
\text { - El paciente no ha tenido un ensayo adecuado con L dopa o un agonista } \\
\text { dopaminérgico. }\end{array}$ \\
\hline $\begin{array}{l}\text { - Criterios para un diagnóstico PROBABLE de EP } \\
\text { Presencia de al menos } 3 \text { de } 4 \text { criterios del grupo A } \\
\text { - Y ninguno de los criterios del grupo B está presente. (Nota: Se necesita una } \\
\text { duración de los síntomas de por lo menos tres años para cumplir este requisito). } \\
\text { - Y se ha documentado una respuesta sustancial y sostenida a L dopa o a una } \\
\text { agonista dopaminérgico. }\end{array}$ \\
\hline $\begin{array}{l}\text { - Criterios para un diagnóstico DEFINITIVO de EP } \\
\text { Se cumplen todos los criterios para un diagnóstico posible de EP. } \\
\text { Y la confirmación histopatológica se obtiene en autopsia. }\end{array}$ \\
\hline $\begin{array}{l}\text { - Criterios histopatológicos para EP } \\
\text { Depleción neuronal sustancial con gliosis acompañanate en la sustancia nigra. } \\
\text { - Por lo menos un cuerpo de Lewy en la sustancia nigra o en el locus coeruleus } \\
\text { (Nota: Es necesario examinar } 4 \text { secciones en cada una de estas áreas antes de } \\
\text { concluir que hay ausencia de cuerpos de Lewy). } \\
\text { - No hay evidencia patológica de otras enfermedades que producen parkinso- } \\
\text { nismo (Parálisis supranuclear progresiva, atrofia multisistémica, degener- } \\
\text { ación gangliónica corticobasal). }\end{array}$ \\
\hline
\end{tabular}

la biblioteca de la Fundación Cardioinfatil - Instituto de Cardiología y se obtuvieron 26 artículos con los resúmenes correspondientes, éstos se analizaron y se seleccionaron 10 artículos, que respondían a las necesidades del estudio, teniendo en cuenta los criterios de inclusión (Tabla 2). Se revisaron también las referencias de cada artículo.

\section{Extracción y síntesis de los datos}

Finalmente se incluyeron en el análisis seis artículos (Figura 1) que cumplían con los criterios de inclusión (Tabla 2). Se excluyeron tres artículos de estudios realizados en 
Tabla 2. Criterios de inclusión y exclusión para la elección de los artículos.

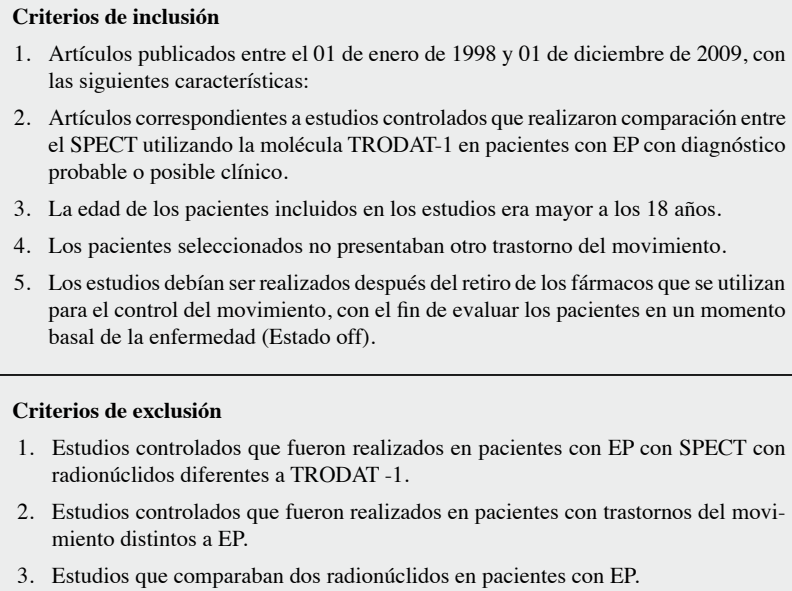

1. Artículos publicados entre el 01 de enero de 1998 y 01 de diciembre de 2009, con las siguientes características:

2. Artículos correspondientes a estudios controlados que realizaron comparación entre el SPECT utilizando la molécula TRODAT-1 en pacientes con EP con diagnóstico probable o posible clínico.

3. La edad de los pacientes incluidos en los estudios era mayor a los 18 años.

4. Los pacientes seleccionados no presentaban otro trastorno del movimiento.

5. Los estudios debían ser realizados después del retiro de los fármacos que se utilizan para el control del movimiento, con el fin de evaluar los pacientes en un momento basal de la enfermedad (Estado off).

Criterios de exclusión

1. Estudios controlados que fueron realizados en pacientes con EP con SPECT con radionúclidos diferentes a TRODAT -1 .

2. Estudios controlados que fueron realizados en pacientes con trastornos del movimiento distintos a EP

3. Estudios que comparaban dos radionúclidos en pacientes con EP

pacientes con EP y radionúclidos diferentes a TRODAT-1, pacientes con desórdenes del movimiento distintos a EP y estudios que comparaban dos fármacos distintos y un artículo que no pudo ser obtenido en texto completo a pesar de los esfuerzos del grupo de investigadores. Todos los artículos fueron leídos y evaluados en forma independiente por dos evaluadores, y las discrepancias fueron resueltas por un tercer evaluador. Se obtuvo la siguiente información de cada artículo: criterios para el diagnóstico de la EP, edad de los pacientes que participan en cada estudio, realización de la prueba (tiempo de evaluación posterior a la administración del TRODAT-1). Resultados de la prueba e interpretación de la misma, sensibilidad y especificidad.

La validez de cada artículo fue evaluada teniendo en cuenta las siguientes características: métodos de asignación de los pacientes, método de realización de la prueba con TRODAT-1, definiciones de diagnóstico y positividad de la prueba, resultados y nivel de calidad de acuerdo con los criterios definidos en el apéndice $\mathrm{F}$ de las guías de evaluación de información de NICE (Anexo 1). De acuerdo con el cumplimiento de estos criterios, los estudios fueron clasificados en tres categorías relacionadas con el nivel de evidencia y riesgo de sesgo (Recomendado por el manual NICE en el Apéndice J) (Anexo 2) (5).

\section{Apreciación crítica de la evidencia}

El análisis estadístico se realizó después de seleccionar los resúmenes teniendo en cuenta los criterios de inclusión. Se implementó la herramienta de las guías NICE utilizando la plantilla de evaluación para los estudios de pruebas diagnósticas (Anexo 1) que contiene 22 preguntas, las primeras ocho son de tipo cualitativo y gradúan el nivel de evidencia en las siguientes categorías: (++) si la prueba diagnóstica es comparada con un "gold standard" y cumple con la mayoría de los criterios de calidad, (+) si la prueba diagnóstica no es comparada con el "gold standard", pero tiene en cuenta
Figura 1. Diagrama de flujo de selección de artículos

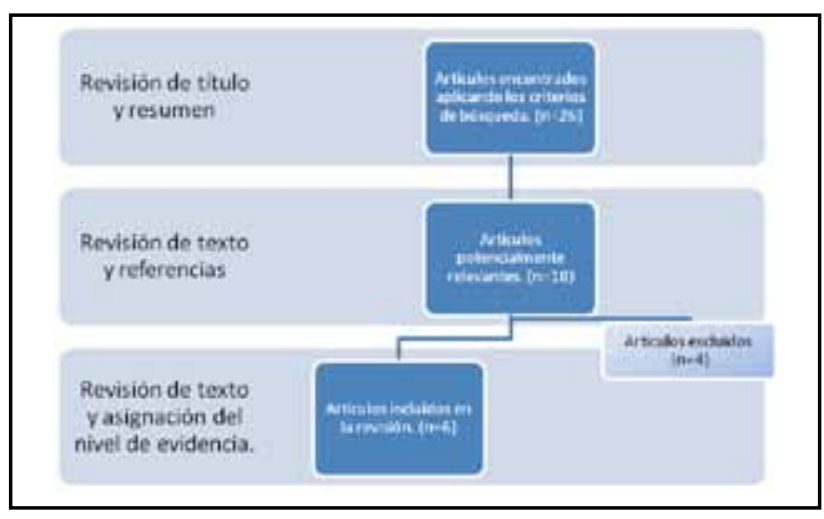

un test de referencia validado y cumple con la mayoría de los criterios de calidad y (-) si el estudio realizado es retrospectivo, y no cumple con la mayoría de los criterios de calidad (Anexo 2).

En este caso, el "gold standard" es el estudio histopatológico, y es muy difícil realizar la comparación con el mismo en el escenario clínico, por lo tanto, ninguno de los estudios revisados tiene un nivel de evidencia correspondiente a (++). Todos realizan la comparación con un test de referencia validado que en este caso es el criterio diagnóstico clínico que según algunos estudios puede alcanzar una exactitud diagnóstica del 90\% (6).

De los seis artículos revisados, cuatro tienen un nivel de evidencia correspondiente a (+) y sólo dos tienen un nivel de evidencia correspondiente a (-), ya que eran de baja calidad metodológica (Tabla 3). Durante el proceso de selección y análisis de los artículos, se encontró que la información contenida en éstos fue insuficiente para realizar un metaanálisis debido a la ausencia de datos esenciales en tres de los estudios para realizar una comparación validera. Se decide, por lo tanto, realizar una revisión sistemática del tema.

Según la guía de NICE, las conclusiones de los estudios calificados con (+) tienen una probabilidad baja de ser alteradas por estudios realizados posteriormente, mientras que los dos estudios calificados con (-) tienen baja validez científica y sus resultados pueden ser alterados por estudios realizados con mejor diseño metodológico.

Todos los artículos comparan el TRODAT-1 SPECT con el criterio clínico diagnóstico, que es el mejor test de referencia ante la imposibilidad de realizar un estudio histopatológico como "gold standard" y sólo uno de ellos realiza de forma adicional una prueba de concordancia entre dos evaluadores (7).

De los seis artículos revisados, sólo uno (8) no menciona los criterios clínicos utilizados para realizar el diagnóstico de EP en la población estudiada. Los demás mencionan los criterios clínicos utilizados por los neurólogos responsables de escoger a los pacientes, basados en el artículo de Gelb et al (1) sobre los criterios clínicos para EP.

En la mayoría de estudios se escogieron pacientes con EP en estadios I y II de la escala de Hoehn y Yahr (HYS) 
Tabla 3. Asignación del nivel de evidencia para los artículos incluidos en la revisión.

\begin{tabular}{|c|c|c|c|c|c|c|c|c|c|}
\hline Referencia bibliográfica & $\begin{array}{c}\text { Nivel de } \\
\text { evidencia }\end{array}$ & $\begin{array}{l}\text { Número de } \\
\text { pacientes }\end{array}$ & Prevalencia & $\begin{array}{c}\text { Características } \\
\text { de los } \\
\text { pacientes }\end{array}$ & $\begin{array}{l}\text { Tipo de } \\
\text { prueba }\end{array}$ & $\begin{array}{l}\text { Estándar } \\
\text { de } \\
\text { referencia }\end{array}$ & $\begin{array}{l}\text { Sensibilidad y } \\
\text { Especificidad }\end{array}$ & $\begin{array}{l}\text { Valores } \\
\text { predictivos } \\
\text { positivo y } \\
\text { negativo }\end{array}$ & Financiación \\
\hline $\begin{array}{l}\text { Weng YH, Yen TC, et al. } \\
\text { J Nucl Med. } 2004 \\
\text { Mar;45(3):393-401. }\end{array}$ & $(+)$ & 78 & No reportada. & $\begin{aligned} & (\mathrm{H}) 4 \\
& (\mathrm{M}) 38 \\
* & (51 \text { a } 77)\end{aligned}$ & $\begin{array}{l}\text { TRODAT -1 } \\
\text { SPECT }\end{array}$ & $\begin{array}{l}\text { Diagnóstico } \\
\text { clínico }\end{array}$ & $100 \%-100 \%$ & $100 \%-100 \%$ & $\begin{array}{l}\text { The Chang-Gung } \\
\text { Medical Research } \\
\text { Council. } \\
\text { The institute of Nuclear } \\
\text { Energy Research. } \\
\text { National Science Council } \\
\text { of Taiwan. }\end{array}$ \\
\hline $\begin{array}{l}\text { Geng Y, Shi GH, et al. } \\
\text { J Zhejiang Univ Sci B. } \\
\text { 2005; 6: 22-7. }\end{array}$ & $(-)$ & 38 & No reportada. & $\begin{array}{c}(\mathrm{H}) 20 \\
(\mathrm{M}) 18 \\
*(31 \text { a } 72)\end{array}$ & $\begin{array}{l}\text { TRODAT -1 } \\
\text { SPECT }\end{array}$ & $\begin{array}{l}\text { Diagnóstico } \\
\text { clínico } \\
\text {. }\end{array}$ & $94 \%-100 \%$ & $100 \%-90 \%$ & No reportada. \\
\hline $\begin{array}{l}\text { Chou KL, Hurtig HI,et al. } \\
\text { Parkinsonism Relat Disord. } \\
\text { 2004;10(6): 375-9. }\end{array}$ & $(+)$ & 29 & No reportada. & $\begin{array}{c}(\mathrm{H}) 20 \\
\text { (M) } 9 \\
*(39 \text { a } 75)\end{array}$ & $\begin{array}{l}\text { TRODAT -1 } \\
\text { SPECT }\end{array}$ & $\begin{array}{l}\text { Diagnóstico } \\
\text { clínico }\end{array}$ & $.79 \%-92 \%$ & $91 \%-19 \%$ & $\begin{array}{l}\text { National institute on Aging } \\
\text { Agency for healthcare } \\
\text { research and quality } \\
\text { ThomasB. McCabe and } \\
\text { Jeanette E. Laws } \\
\text { McCabe Fund. }\end{array}$ \\
\hline $\begin{array}{l}\text { Huang WS, Lin SZ, et al. } \\
\text { J Nucl Med 2001;42: } \\
\text { 1303-1308. }\end{array}$ & $(+)$ & 34 & No reportada. & $\begin{array}{c}(\mathrm{H}) 17 \\
(\mathrm{M}) 17 \\
*(47 \text { a } 79)\end{array}$ & $\begin{array}{l}\text { TRODAT -1 } \\
\text { SPECT }\end{array}$ & $\begin{array}{l}\text { Diagnóstico. } \\
\text { clínico }\end{array}$ & No reportada. & No reportada. & $\begin{array}{l}\text { Atomic Energy Council } \\
\text { The National Science } \\
\text { Council } \\
\text { The Ministry of } \\
\text { Economical Affairs } \\
\text { Republic of China. }\end{array}$ \\
\hline $\begin{array}{l}\text { Huang WS, Lee MS, et al. } \\
\text { Eur J Nucl Med Mol. } \\
\text { Imaging 2004;31(2):155-61. }\end{array}$ & $(+)$ & 188 & No reportada. & $\begin{array}{c}(\mathrm{H}) 103 \\
(\mathrm{M}) 85 \\
*(47 \mathrm{a} 80)\end{array}$ & $\begin{array}{l}\text { TRODAT -1 } \\
\text { SPECT }\end{array}$ & $\begin{array}{l}\text { Diagnóstico } \\
\text { clínico } \\
\text {. }\end{array}$ & $98 \%-86 \%$ & No reportada. & No reportada. \\
\hline $\begin{array}{l}\text { Mena I, Díaz F, et al. } \\
\text { Alasbbimn(Alasbimn) } \\
\text { Journal 2001; 3(10). }\end{array}$ & $(-)$ & 20 & No reportada. & $\begin{array}{c}(\mathrm{H}) 15 \\
(\mathrm{M}) 5 \\
*(24 \mathrm{a} 73 \mathrm{a})\end{array}$ & $\begin{array}{l}\text { TRODAT -1 } \\
\text { SPECT }\end{array}$ & $\begin{array}{l}\text { Diagnóstico } \\
\text { clínico }\end{array}$ & $90 \%-70 \%$ & No reportada & $\begin{array}{l}\text { Research programs } \\
\text { collaboration between } \\
\text { the Institute of Nuclear } \\
\text { Energy Research y } \\
\text { Clínica Las Condes - } \\
\text { Santiago de Chile. }\end{array}$ \\
\hline
\end{tabular}

y sólo uno evalua pacientes con EP con estadios severos de la enfermedad (HYS I a V) (7), que es también el estudio con mayor cantidad de pacientes.

En cuanto a los criterios de inclusión además de tener en cuenta el diagnóstico clínico de EP, sólo uno tuvo en cuenta otros criterios de inclusión en cuanto a las mujeres que quisieran participar en el estudio relacionado con la seguridad de no estar en gestación en el momento de la prueba (9). Cinco de los estudios suspendieron los medicamentos antiparkinsonianos por lo menos 12 horas antes de la prueba para evitar un posible sesgo, aunque en experimentos realizados en ratas las dosis de medicamentos utilizados normalmente parecen no afectar el pool de neurotransmisores que el SPECT pretende evaluar (7).

En cuanto a la cantidad de pacientes estudiados, el estudio con menos pacientes evalúa 20 y el mayor evalúa 188 , se observa también que todos involucran pacientes mayores de 35 o 40 años, que es el tipo de población en el cual el diagnóstico temprano de EP puede ser de mayor valor, hasta los 80 años, que es la edad en la cual se aumenta la incidencia de la enfermedad. Todos seleccionan más hombres que mujeres afectados por EP, lo cual puede corresponder a la mayor prevalencia de la enfermedad en hombres.

Los criterios de inclusión para los controles en los diferentes estudios es (son) homogéneo(s) y se trata de per- sonas sin antecedentes de enfermedades neuropsiquiátricas, historia de consumo de sustancias con efectos en el sistema nervioso central, cirugías neurológicas y de la misma edad y género.

Todos los estudios utilizan gammacámaras de doble cabezal, excepto uno que utiliza gammacámara de triple cabezal (9), y las imágenes son obtenidas 3 a 4 horas después de la inyección del radiotrazador, en todos los artículos, comparándolas con imágenes de resonancia magnética.

La forma de evaluar las imágenes obtenidas en los artículos es diferente en todos. El trabajo de Weng et al (10), describe una forma semicuantitativa de realizar la medición de la captación del radiofármaco en los cuerpos estriados de acuerdo con unas fórmulas creadas para establecer proporciones de captación en comparación con la corteza occipital.

El trabajo de Geng et al propone una fórmula diferente para establecer una proporción de cuentas en comparación con la corteza occipital. El trabajo de Chou KL et al (9) establece fórmulas parecidas pero utiliza como punto de comparación las cortezas frontal y parietal. El estudio de Huang et al (11) evalúa la captación con fórmulas similares a las utilizadas por Geng, sin embargo en un segundo trabajo realizado por el mismo autor (7) la escala utilizada para realizar las mediciones de sensibilidad y especificidad es 
una escala visual dividida en dos partes "fina" y "burda", además de utilizar la escala semicuantitativa mencionada previamente. Por último el estudio de Mena I et al (8) utiliza una escala semicuantitativa en comparación con la corteza occipital.

La utilización de las escalas visuales propuesta representa una forma fácil de evaluar los resultados de la prueba con un buen nivel de concordancia entre los evaluadores de acuerdo con el artículo de Huang WS et al (7).

Sólo tres de los artículos estudiados $(9,10,12)$ reportan sensibilidad y especifidad para cada núcleo estudiado (Estriado, núcleo caudado, putamen en sus porciones anterior y posterior), los resultados publicados en la (Tabla 3) son los obtenidos a nivel del putamen contralateral en cada estudio. Aunque los demás utilizan técnicas semicuantitativas no proponen un punto de corte y no se menciona en forma explícita cuáles son los verdaderos positivos, verdaderos negativos, falsos positivos y falsos negativos para realizar las tablas de $4 \times 4$.

Uno de los artículos (9) propone un valor predictivo positivo de $91 \%$, ofreciendo una probabilidad de tener la enfermedad en una persona con una probabilidad pre test de $50 \%$ para EP.

Todos los artículos mencionan como principal hallazgo una disminución de la captación del radiofármaco en el putamen posterior contralateral, siendo este un hallazgo importante para realizar un diagnóstico diferencial con otros parkinsonismos.

El único estudio que incluye pacientes con EP en fases avanzadas (7), reporta pérdida de la captación en todo el estríado.

Todos concuerdan en la posible utilidad del TRODAT -1 para instaurar el diagnóstico de la EP, incluso previo a la aparición de los síntomas motores y en el seguimiento de los mismos, porque algunos se corrrelacionan con el grado de severidad de la enfermedad.

Todos concuerdan en que la técnica SPECT es de bajo costo, puede ser utilizada ampliamente y no necesita ciclotrón, es una técnica segura.

\section{Conclusiones}

El objetivo de esta revisión es determinar la utilidad del TRODAT-1 en el diagnóstico de la EP, sin embargo por la calidad de los estudios, y debido a que sólo tres de ellos reportan valores de sensibilidad y especificidad, obtenidos a través de técnicas distintas de evaluación, no es posible realizar un metaanálisis. La prueba diagnóstica no presenta diferencias significativas en cuanto a su realización, el radiofármaco utilizado es el mismo; el tiempo de obtención de las imágenes después de la administración del radiofármaco es similar y concuerda con otros reportes obtenidos previamente. La importancia de la utilización de gammacámaras de doble o triple cabezal, no es mencionada ampliamente en estos artículos, excepto en uno (7), que plantea la posibilidad de mejorar la calidad de la imagen con el uso de gammacá- maras de triple cabezal que permitirían obtener una mejor resolución de imagen con una mejor diferenciación de los núcleos que conforman el estríado, lo cual facilitaría la técnica para dibujar el área de interés ( ROI) en cada uno y tener así una mejor evaluación semicuantitativa.

La gran diferencia y mayor punto de discusión es la herramienta empleada por cada grupo investigador en la evaluación de la prueba. No hay un consenso acerca de las medidas que deberían realizarse para medir la captación, y tampoco con cual porción de la corteza realizar la comparación. La mayoría de los estudios realizan una comparación con la corteza occipital debido a que ésta ha demostrado tener un nivel de captación del radiofármaco inespecífica, sin embargo, en el estudio realizado por Chou KL et al (9) la comparación es realizada con la corteza frontoparietal que tiene también áreas de captación inespecífica y según ellos no tiene una captación tan baja para producir una medida errónea.

Tres de los artículos $(7,10,11)$ proponen las mismas ecuaciones para realizar la medición de las SUV (Mean specific uptake values) o unidades de captación específica. Para realizar esta medición el evaluador de la imagen debe dibujar un ROI (Region of interest) sobre cada uno de los núcleos que se pretende evaluar, si el estudio no es ciego, pueden generarse sesgos en cuanto a la forma de realizar la asignación del ROI, siendo esta variable operador dependiente. Una vez asignado el ROI, el equipo suministra la información sobre las cuentas por píxel y las SUV, pero si se quiere comparar con una región de la corteza con captación inespecífica deben crearse unas ecuaciones para realizar el cálculo. Todos los artículos proponen ecuaciones que pueden ser similares, pero no hay un consenso de cómo hacer la medición, o cuál es la mejor ecuación para hacerlo. Por ejemplo, en los artículos de Weng YH y Huang WS (7, 10, 11) la ecuación utilizada para calcular la captación en determinado núcleo es la siguiente: [(Cuentas en el ROI - cuentas en la corteza occipital)]/ (Cuentas en la corteza occipital); pero en el estudio de Geng et al (12) la ecuación utilizada es la siguiente: [Cuentas por píxel en el núcleo / (Cuentas por píxel en el cerebelo + Cuentas por píxel en la corteza occipital/2)], por esto aunque todos pretenden realizar una medición semicuantitativa de la actividad del radiofármaco en los puntos de interés debe proponerse un consenso para conseguir la misma medición y permitir la comparación de estos resultados.

Uno de los hallazgos más importantes y que fue constante en los estudios fue la pérdida temprana de captación en la parte posterior del putamen contralateral a los síntomas, que ha sido reportada previamente, todos los artículos mencionaron este hallazgo y algunos realizaron la propuesta de tomarlo como una característica que podría ayudar a establecer el diagnóstico diferencial e indicador de EP temprana, mencionando que esto no se encuentra en otras formas de parkinsonismo. El hallazgo temprano de esta característica en la EP incluso antes de la aparición 
de algunos de los síntomas motores es lo que hace más útil e interesante la prueba, porque es precisamente en la población con EP temprana en la que el diagnóstico precoz ayudaría a establecer un tratamiento inicial enfocado a la enfermedad y en el futuro, la posibilidad de utilizar una terapia neuroprotectora, disminuyendo la morbilidad de la enfermedad. Esta afirmación está presente en todos los artículos, además de proporcionar información importante acerca de la fisiopatología de la EP en la cual la pérdida neuronal dopaminérgica se realiza en un gradiente de posterior hacia anterior en el putamen y en estadios más avanzados compromete el núcleo caudado y la totalidad del cuerpo estriado. Este hallazgo es reportado por Huang WS, (7) que fue el único artículo que realizó imágenes en pacientes con EP severa.

La técnica de TRODAT-1 no reportó efectos adversos en ninguno de los pacientes incluidos en los estudios, demostrando ser una técnica segura, que debe ser utilizada de acuerdo con las normas de radioprotección establecidas en los departamentos de medicina nuclear a nivel mundial.

Por último, todos los artículos están de acuerdo con la posibilidad de realizar esta prueba de una forma global, debido al bajo costo, comparado con otras técnicas de medicina nuclear que involucran la función de la sinapsis dopaminérgica y requieren radiofármacos creados en ciclotrón que aumentaría su valor (costo) considerablemente. El TRODAT-1 sólo necesita ser marcado con TC99m, y esto lo hace una técnica de fácil acceso y bajo costo que puede ser utilizada de forma segura para el diagnóstico de la EP, en especial en la población con EP temprana.

\section{Agradecimientos}

Agradecemos al Dr. Rodolfo Dennis, director de la oficina de investigaciones de la Fundación Cardioinfantil - Instituto de Cardiología, por la revisión previa de este manuscrito.

\section{Referencias}

1. Gelb DJ, Oliver E, Gilman S. Diagnostic criteria for Parkinson disease. Arch Neurol 1999; 56: 33-9.

2. Chi Shih M, Queiroz M, Franco LA, Affonseca R. Parkinson's disease and dopamine transporter neuroimaging - a critical review. Sao Paulo Med J 2006; 124: $168-75$.

3. Hammoud D, Hoffman M, Pomper M. Molecular neuroimaging: From conventional to emerging techniques. Radiology 2007; 24: 21-42.

4. Kung H, Kung M, Wey S, Lin K, Yen T. Clinical acceptance of a molecular imaging agent: a long march with [ $\left.{ }^{99 \mathrm{~m}} \mathrm{Tc}\right]$ TRODAT-1. Nuclear and medicine biology 2007; 34: 787-89.

5. National Institute for Clinical Excellence (February 2004, updated 2005) Guideline Development Methods: Information for National Collaborating Centres and Guideline Developers. London: National Institute for Clinical Excellence.

6. Hughes AJ, Daniel SE, Ben - Shlomo Y, Lees AJ. The accuracy of diagnosis of parkinsonian síndromes in a specialist movement disorder service. Brain 2002; 125: 861-70.

7. Huang WS, Lee MS, Lin JC, Chen CY, Yang YW, Lin SZ, et al. Usefulness of brain 99mTc-TRODAT-1 SPET for the evaluation of Parkinson's disease. Eur J Nucl Med Mol Imaging 2004; 31:155-61.

8. Mena I, Díaz F, Gómez A. Diagnóstico precoz de enfermedad de Parkinson: NeuroSPECT mediante TRODAT -1 Tc 99m, marcador del transportador de dopamina. Alasbbimn (Alasbimn) Journal 2001; 3: 10.

9. Chou KL, Hurtig HI, Stern MB, Colcher A, Ravina B, Newberg A, et al. Diagnostic accuracy of [99mTc]TRODAT-1 SPECT imaging in early Parkinson's disease. Parkinsonism Relat Disord 2004; 10: 375-9.

10. Weng YH, Yen TC, Chen MC, Kao PF, Tzen KY, Chen RS, et al. Sensitivity and specificity of 99mTc-TRODAT-1 SPECT imaging in differentiating patients with idiopathic Parkinson's disease from healthy subjects. J Nucl Med 2004; 45: 393-401.

11. Huang WS, Lin SZ, Lin JC, Wey SP, Ting G, Liu RS. Evaluation of early stage Parkinson disease with 99mTC TRODAT-1 imaging. J Nucl Med 2001; 42: $1303-8$.

12.Geng Y, Shi GH, Jiang Y, Xu LX, Hu XY, Shao YQ. Investigating the role of 99mTc-TRODAT-1 SPECT imaging in idiopathic Parkinson's disease. $J$ Zhejiang Univ Sci B 2005; 6: 22-7. 


\section{ANEXO 1. PLANTILLA DE EVALUACIÓN PARA PRUEBAS DIAGNÓSTICAS (5).}

Guías NICE: Apéndice F

Lista de comprobación: pruebas diagnósticas.

\begin{tabular}{|c|c|c|c|}
\hline \multicolumn{4}{|c|}{ Identificación del estudio: Incluye autor, título, referencia, año de publicación. } \\
\hline \multicolumn{4}{|c|}{ SECCIÓN 1: VALIDEZ INTERNA } \\
\hline \multicolumn{2}{|c|}{ En una prueba diagnóstica } & \multicolumn{2}{|c|}{$\begin{array}{c}\text { En este estudio el criterio es: } \\
\text { (Marque con un círculo para cada pregunta) }\end{array}$} \\
\hline 1.1 & La naturaleza de la prueba en estudio se encuentra claramente especificada. & $\begin{array}{l}\text { Bien cubierto } \\
\text { Adecuadamente dirigido } \\
\text { Pobremente dirigido }\end{array}$ & $\begin{array}{l}\text { No dirigido } \\
\text { No reportado } \\
\text { No aplica }\end{array}$ \\
\hline 1.2 & La prueba es comparada con el "gold standard” adecuado. & $\begin{array}{l}\text { Bien cubierto } \\
\text { Adecuadamente dirigido } \\
\text { Pobremente dirigido }\end{array}$ & $\begin{array}{l}\text { No dirigido } \\
\text { No reportado } \\
\text { No aplica }\end{array}$ \\
\hline 1.3 & $\begin{array}{l}\text { Si no existe un "gold standard", un estándar de referencia válido ha sido utilizado } \\
\text { como comparación. }\end{array}$ & $\begin{array}{l}\text { Bien cubierto } \\
\text { Adecuadamente dirigido } \\
\text { Pobremente dirigido }\end{array}$ & $\begin{array}{l}\text { No dirigido } \\
\text { No reportado } \\
\text { No aplica }\end{array}$ \\
\hline 1.4 & $\begin{array}{l}\text { Los pacientes para la prueba han sido seleccionados en forma consecutiva o aleatoriamente } \\
\text { de una población de estudio claramente definida. }\end{array}$ & $\begin{array}{l}\text { Bien cubierto } \\
\text { Adecuadamente dirigido } \\
\text { Pobremente dirigido }\end{array}$ & $\begin{array}{l}\text { No dirigido } \\
\text { No reportado } \\
\text { No aplica }\end{array}$ \\
\hline 1.5 & La prueba y el "gold standard" son medidos de forma independiente (ciega) uno del otro. & $\begin{array}{l}\text { Bien cubierto } \\
\text { Adecuadamente dirigido } \\
\text { Pobremente dirigido }\end{array}$ & $\begin{array}{l}\text { No dirigido } \\
\text { No reportado } \\
\text { No aplica }\end{array}$ \\
\hline 1.6 & La prueba y el "gold standard" son aplicados tan cercanamente como es posible. & $\begin{array}{l}\text { Bien cubierto } \\
\text { Adecuadamente dirigido } \\
\text { Pobremente dirigido }\end{array}$ & $\begin{array}{l}\text { No dirigido } \\
\text { No reportado } \\
\text { No aplica }\end{array}$ \\
\hline 1.7 & Los resultados son reportados para todos los pacientes que entraron al estudio. & $\begin{array}{l}\text { Bien cubierto } \\
\text { Adecuadamente dirigido } \\
\text { Pobremente dirigido }\end{array}$ & $\begin{array}{l}\text { No dirigido } \\
\text { No reportado } \\
\text { No aplica }\end{array}$ \\
\hline $\begin{array}{ll}\text { EVAI } \\
1.8\end{array}$ & $\begin{array}{l}\text { LUACIÓN } \\
\text { Un prediagnóstico es realizado y reportado. }\end{array}$ & $\begin{array}{l}\text { Bien cubierto } \\
\text { Adecuadamente dirigido } \\
\text { Pobremente dirigido }\end{array}$ & $\begin{array}{l}\text { No dirigido } \\
\text { No reportado } \\
\text { No aplica }\end{array}$ \\
\hline \multicolumn{4}{|c|}{ SECCIÓN 2: EVALUACIÓN TOTAL DEL ESTUDIO } \\
\hline 2.1 & ¿Qué tan confiables son las conclusiones del estudio? Codifique como(++),(+) ० (-). & $(++)$ & $(-)$ \\
\hline 2.2 & $\begin{array}{l}\text { ¿El espectro de pacientes evaluados en este estudio es comparable con el grupo de } \\
\text { pacientes blanco, en términos de proporción de la enfermedad o la proporción entre } \\
\text { enfermedad severa versus enfermedad leve? }\end{array}$ & & \\
\hline 3.8 & ¿Cuál es el valor predictivo positivo de la prueba diagnóstica evaluada? & & \\
\hline 3.9 & ¿Cuál es el valor predictivo negativo de la prueba diagnóstica evaluada? & & \\
\hline 3.10 & Cuáles son las proporciones de probabilidad para la prueba diagnóstica evaluada? & & \\
\hline 3.11 & ¿Cómo fue financiado este estudio? & & \\
\hline 3.12 & $\begin{array}{l}\text { ¿Hay alguna conclusión que pueda ser obtenida a partir de este estudio? } \\
\text { ¿Cómo ayuda este estudio a responder su pregunta? }\end{array}$ & & \\
\hline
\end{tabular}

ANEXO 2. NIVELES DE EVIDENCIA SEGÚN LAS GUÍAS NICE (5).

\begin{tabular}{|c|l|}
\hline Nivel de evidencia & \multicolumn{1}{c|}{ Descripción del código utilizado } \\
\hline$(++)$ & $\begin{array}{l}\text { El estudio es prospectivo y compara la prueba diagnóstica con el "gold standard" y los criterios de calidad se encuentran en su mayoría adecua- } \\
\text { damente dirigidos. Es muy poco probable que las conclusiones del estudio sean alteradas en futuros estudios. }\end{array}$ \\
\hline$(+)$ & $\begin{array}{l}\text { El estudio es prospectivo y compara la prueba diagnóstica con una prueba de referencia validada y los criterios de calidad se encuentran en su } \\
\text { mayoría adecuadamente dirigidos. Es poco probable que las conclusiones del estudio sean alteradas en futuros estudios. }\end{array}$ \\
\hline$(-)$ & $\begin{array}{l}\text { El estudio es retrospectivo o falla en dirigir adecuadamente la mayoría de los criterios de calidad. Estudios mejor diseñados probablemente } \\
\text { alterarán las conclusiones. }\end{array}$ \\
\hline
\end{tabular}

